\title{
Médecine pénitentiaire en Valais, minuit moins une!
}

Marc-Henri Gauchat

Président de la Société médicale du Valais, député
Il ne se passe presque pas une semaine sans que la presse ne rapporte sur les prisons valaisannes $[1,2,3]$.

La situation est inquiétante! En effet, le Réseau Santé Valais (RSV), qui avait accepté le mandat de l'Etat du Valais pour prendre en charge la médecine pénitentiaire (Service de médecine pénitentiaireIPVR), a dénoncé ce contrat avec effet au 30 juin 2012. Comment en est-on arrivé à cette situation difficile?

L'audit du système pénitentiaire valaisan a pointé sur un certain nombre de dysfonctionnements et de lacunes dans l'organisation qui ne sont plus compatibles avec les normes en vigueur dans ce secteur [4]. Le SMP-IPVR a cherché à rencontrer la Conseillère d'Etat en charge du dossier à plusieurs reprises, sans succès. Le SMP estime ne plus pouvoir exercer son mandat de manière satisfaisante tant que les conditions restent identiques, notamment en ce qui concerne:

- le manque de personnel de surveillance pour conduire les détenus à la consultation (prison des îles, par ex.),

- le manque de ressources nécessaires pour le traitement des personnes soumis à des mesures d'internement selon art. 53 al. 3 CPS,

- le manque de modèle intégratif et de ressources médicales pour le traitement des jeunes placés à Pramont, lieu de placement éducatif, et qui présentent des souffrances et des troubles mentaux parfois très lourds,

- l'impossibilité d'évaluer et de connaitre l'activité médicale et psychiatrique de l'établissement de Brigue, problèmes confirmés par la Commission des aumôniers des prisons de l’Eglise Réformée,

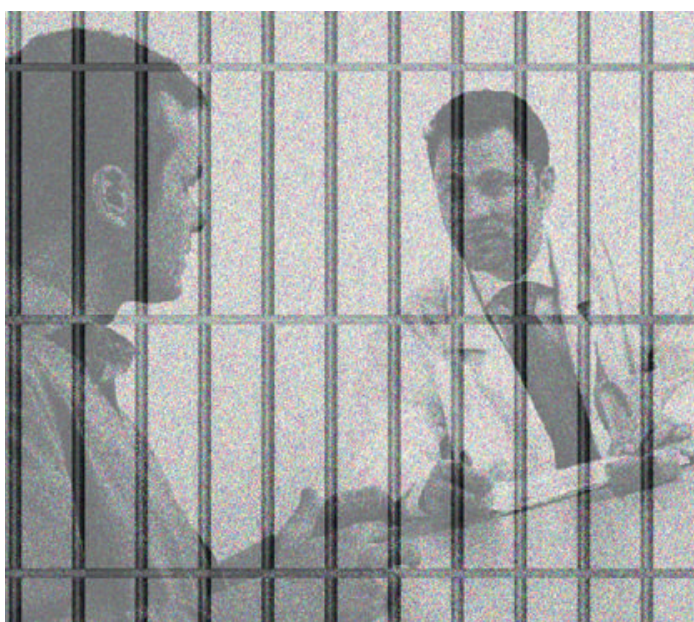

La situation de la médecine pénitentiaire en Valais est inquiétante.
- le mode de fonctionnement très administratif et sanctionnant de la direction des EPV,

- la tentative de mise sous tutelle du SMP par la direction des EPV.

Cette situation est incompatible avec le déploiement d'une activité de médecine pénitentiaire indépendante tel que l'exigent les directives médico-éthiques de l'Académie Suisse des Sciences Médicales (ASSM) dans les établissements pénitentiaires.

Le groupe de travail chargé par le Département de la Sécurité, des Affaires sociales et de l'Intégration (DSSI) de trouver des pistes et des solutions pour un nouveau concept médical ne semble pas encore avoir siégé. Il serait temps que les conditions fixées par le SMP pour la poursuite de son activité soient examinées et discutées, soit:

- que soient levés les obstacles à l'accès aux prestations ou aux soins en dotant les établissements pénitentiaires des ressources nécessaires pour accompagner les détenus ou soignants vers les espaces de consultation médicale,

- que soient clairement définis les périmètres d'action et de responsabilité des soignants avec une dotation suffisante pour y assurer une activité médicale digne de ce nom,

- que la charge financière du SMP soit transférée du DSSI vers le Département des Finances des Institutions et de la Santé DFIS,

- qu'une plateforme interinstitutionnelle de concertation entre le Département de la Sécurité, des Affaires sociales et de l'Intégration DSSI, le DFIS et le RSV soit mise en place immédiatement pour accompagner cette réforme.

La balle est maintenant dans le camp des politiques, et il serait temps d'agir sous peine de se retrouver dans la situation des pays totalitaires avec une médecine des prisons à la botte du pouvoir administratif! [5]

\section{Références}

1 Audit sévère du système carcéral valaisan, ATS, Le Temps, 23 septembre 2011.

2 Georges Sewer, maître contesté des prisons du Valais, Marie Parvex, Le Temps, 11 février 2012.

3 Le Valais fait volte-face sur l'alimentation forcée des détenus, Denis Masmejan, Le Temps, 10.3.2012.

4 Rapport final de l'audit systématique sur le fonctionnement des établissements pénitentiaires valaisans, Benjamin F. Brägger, 2 septembre 2011.

5 La pratique médicale dans les prisons: comparaison internationale, Jean-Pierre Restellini, in Rapports entre médecins et autorités: indépendance ou collaboration? 18 Journée du droit de la Santé, Neuchâtel 2011. 